\title{
NON LINEAR SYSTEMS DESCRIBING PHASE TRANSITION MODELS COMPATIBLE WITH THERMODYNAMICS
}

\author{
Pierluigi COLLI \\ Dipartimento di Matematica "F. Casorati", Università di Pavia, Italy. \\ pier@dragon.ian.pv.cnr.it \\ Giorgio GENTILI \\ Dipartimento di Matematica, Università di Bologna, Italy. \\ gentili@dm.unibo.it \\ Claudio GIORGI \\ Dipartimento di Elettronica per l'Automazione, Università di Brescia, Italy. \\ giorgi@bsing.ing.unibs.it
}

\begin{abstract}
This paper is devoted to analyse solutions of a nonlinear evolution system describing the phase transition in a rigid heat conductor in presence of phase relaxation. First, in a general framework, a rate type constitutive law for the phase variable is considered and matched with the Helmholtz free energy involving the state of the material. Thermodinamic compatibility of the resulting models is scrutinized. Moreover, a comparison with a different phase change modelling is performed. Under proper assumptions, a nonlinear system in the (absolute) temperature and phase variable is achieved. For it, existence and uniqueness of the solution is proved and positivity of temperature is recovered.
\end{abstract}

\section{Introduction}

This paper is concerned with a family of thermodynamically consistent models for thermally induced phase transitions, when deformation and mass diffusion are negligible. The energy balance equation governes the evolution of the temperature $\theta$ and the kinetics of the transition is macroscopically described by a non-conserved phase variable $\chi$. The phase diffusion is neglected by assuming that no interfacial energy occurs. Therefore, a rate-type consitutive law governing the evolution of $\chi$ accounts for phase relaxation, only.

Our purpose is twofold. First, in the framework of phase-relaxation we develop a general thermodynamic theory which turns out to include many different models previously considered as unrelated. For instance, Stefan-type problems with phase relaxation, ${ }^{20}$ standard phase field ${ }^{1,14,24}$ and Penrose-Fife ${ }^{17,18}$ models with zero interfacial energy, and some models with finite thermal memory proposed by Fabrizio and Gentili ${ }^{12}$ enter our general setting. Many of these models have been recently 
considered and their related initial-boundary value problems have been analysed from the mathematical point of view (see, for instance, Refs. 5, 23 and references therein). The second goal of this paper is to study a class of mathematical problems which are related to a special model not yet investigated. In particular, we deal with the system of differential equations when the heat flux is described by the linear Fourier law and the constitutive rate-type law for $\chi$ is given by

$$
F_{2}^{\prime}(\chi) \dot{\chi}=\frac{\partial \psi}{\partial \chi}(\theta, \chi)
$$

where $\psi$ denotes the free energy and $F_{2}$ is a monotone increasing $C^{1}$-function.

The paper is organized as follows. In the next section, we introduce the set of balance and constitutive equations together with the Clausius-Duhem inequality and develop a family of phase-transition models compatible with thermodynamics (Subsection 2.1). Then, we discuss the choice of the free energy in connection with the existence of a mushy region and the presence of superheating and undercooling effects at equilibrium (Subsection 2.2). Finally, we prove that some special choices of the involved constitutive functions lead to well-established phase relaxation and phase field problems (Subsection 2.3).

In Section 3 we introduce a class of initial boundary value problems. In Subsection 3.1, by a suitable mathematical formulation of the problem we state the main theorems about existence and uniqueness of the solution as well as boundedness and positivity of the absolute temperature. These last properties are not needed for the existence proof, nevertheless they appear quite fundamental for the validation of the physical model. The other subsections are devoted to showing the results. In particular, in Subsection 3.2, uniqueness of the solution is derived with the help of an a priori estimate. In Subsection 3.3, we prove existence by using an approximation method and passage to the limit along with compactness and continuity arguments. Finally, in Subsection 3.4, we rigorously check that the solution component $\theta$ (of the pair $(\theta, \chi)$ satisfying the initial boundary value problem) is uniformly bounded and actually attains non-negative values.

\section{Thermodynamically consistent models}

This section is devoted to the analysis of some rigid phase transition models depending on temperature. To this end, we consider a rigid heat conductor occupying a fixed bounded domain. Such a material enters the class of "simple materials" (see Ref. 13) so that the process is given by the pair $(\dot{\theta}, \nabla \theta)^{a}$ " Henceforth, for any $u=u(\mathbf{x}, t), \dot{u}$ denotes the partial derivative of $u$ with respect to time $t, \nabla u$ stands for the gradient (with respect to space variable $\mathbf{x}$ ), whereas, if $f$ is a function of one variable only, $f^{\prime}$ denotes its derivative with respect to the argument. where $\theta>0$ is the absolute temperature. Here we assume the state is described by $(\theta, \chi)$ where $\chi \in[0,1]$ is the phase variable (sometimes called order parameter) which represents the concentration of the more energetic phase and plays the role of an internal variable for the material. 
Our starting assumptions are resumed into the constitutive equations for the internal energy e, the heat flux $\mathbf{q}$ and the phase variable $\chi$. The internal energy is assumed to be the sum of a function of $\theta$ and a function of $\chi$, that is,

$$
e(\theta, \chi)=f_{0}(\theta)+f_{1}(\chi) .
$$

Usual properties of the internal energy impose the specific heat to be positive, namely,

$$
c_{v}=\frac{\partial e}{\partial \theta}>0 .
$$

Moreover, by virtue of the meaning of the phase variable (we remind that $\chi=1$ represents the more energetic phase density), the energy must increase with $\chi$. The above assumptions justify the following relations

$$
f_{0}^{\prime}(\theta)>0 \quad, \quad f_{1}^{\prime}(\chi) \geq 0 .
$$

For later convenience, in the sequel we use two suitable $C^{1}$ functions $F_{0}$ and $F_{1}$ such that $f_{0}(\theta)=\theta^{2} F_{0}^{\prime}(\theta)$ and $f_{1}(\chi)=\theta_{c} F_{1}(\chi)$, where $\theta_{c}$ denotes a critical reference temperature around which the transition should occur. Thus, we have

$$
e(\theta, \chi)=\theta^{2} F_{0}^{\prime}(\theta)+\theta_{c} F_{1}(\chi) .
$$

The heat flux vector q is supposed to obey Fourier's law, that is,

$$
\mathbf{q}=-\kappa(\theta) \nabla \theta .
$$

Finally, we assume that the gradient of the phase variable does not contribute to the evolution of the material at all, so that no interfacial energy occurs. More precisely, we take a "rate type" constitutive equation for $\chi$ of the general form

$$
\dot{\chi}=\gamma(\theta, \chi) \text {. }
$$

The constitutive functions $F_{0}, F_{1}, \kappa$ and $\gamma$ cannot be arbitrarily chosen, but they have to satisfy the Second Principle of Thermodynamics, as we discuss next.

\subsection{Balance equations, free energy and thermodynamic restrictions}

The evolution of a rigid phase change phenomenon is governed by the energy balance equation as

$$
\dot{e}+\operatorname{div} \mathbf{q}=r,
$$

where $r$ is the heat source density. Physically admissible processes are all solutions of (2.6) satisfying the Second Principle of Thermodynamics, that is here stated by the Clausius-Duhem inequality

$$
\dot{\psi}+\eta \dot{\theta}+\frac{\mathbf{q} \cdot \nabla \theta}{\theta^{2}} \leq 0,
$$


where the free energy $\psi(\theta, \chi)$ and the entropy $\eta(\theta, \chi)$ are state functions, related to the internal energy $e$ by the standard relation

$$
e=\psi+\theta \eta \text {. }
$$

Thanks to (2.4) and (2.5), as $\dot{\psi}=\frac{\partial \psi}{\partial \theta} \dot{\theta}+\frac{\partial \psi}{\partial \chi} \dot{\chi}$ then (2.7) becomes

$$
\left(\frac{\partial \psi}{\partial \theta}+\eta\right) \dot{\theta}+\frac{\partial \psi}{\partial \chi} \gamma-\frac{\kappa|\nabla \theta|^{2}}{\theta^{2}} \leq 0 .
$$

Since the heat supply $r$ in (2.6) allows us to choose the value of the process $(\dot{\theta}, \nabla \theta)$ independently of the state $(\theta, \chi)$, inequality $(2.9)$ implies the following conditions

$$
\eta(\theta, \chi)=-\frac{\partial \psi}{\partial \theta}(\theta, \chi) \quad, \quad \kappa(\theta) \geq 0 \quad, \quad \frac{\partial \psi}{\partial \chi}(\theta, \chi) \gamma(\theta, \chi) \leq 0 .
$$

Inequalities $(2.10)_{2}$ and $(2.10)_{3}$ have the meaning of thermodynamic restrictions on the constitutive equations (2.4) and (2.5), respectively, whereas $(2.10)_{1}$ can be used to find a general expression of the free energy $\psi$ according to (2.1). In view of (2.8), $(2.10)_{1}$ and (2.3), a thermodinamically compatible form of $\psi$ can be deduced by solving the differential equation

$$
\psi-\theta \frac{\partial \psi}{\partial \theta}=\theta^{2} F_{0}^{\prime}(\theta)+\theta_{c} F_{1}(\chi) .
$$

A straightforward calculation leads to

$$
\psi(\theta, \chi)=-\theta F_{0}(\theta)+\theta B(\chi)-\left(\theta-\theta_{c}\right) F_{1}(\chi),
$$

where $B$ is an arbitrary function whose choice will be scrutinized later.

We conclude this subsection by discussing a particular setup for the rate-type constitutive equation (2.5) in agreement with the thermodynamic restriction $(2.10)_{3}$. In order to satisfy $(2.10)_{3}$, henceforth we assume

$$
\gamma(\theta, \chi)=-\frac{1}{g(\theta, \chi)} \frac{\partial \psi}{\partial \chi}(\theta, \chi) \quad \text { where } \quad g(\theta, \chi)>0 \quad \forall(\theta, \chi) .
$$

According to the viewpoint of Frémond and Visintin, ${ }^{15}$ (2.12) relates the free energy $\psi$ to a suitable dissipation pseudopotential $U(\theta, \chi, \dot{\chi})$, which turns out to be convex and quadratic with respect to $\dot{\chi}$. In fact, postulating that

$$
g(\theta, \chi) \dot{\chi}=\frac{\partial U}{\partial \dot{\chi}}(\theta, \chi, \dot{\chi}),
$$

constitutive equation (2.5) can be rewritten as

$$
\frac{\partial \psi}{\partial \chi}(\theta, \chi)+\frac{\partial U}{\partial \dot{\chi}}(\theta, \chi, \dot{\chi})=0 .
$$


For the sake of simplicity, from now on we let

$$
g(\theta, \chi)=F_{2}^{\prime}(\chi) N(\theta) .
$$

According to $(2.12), N(\theta)$ is required to be a continuous, positive function and $F_{2}$ a monotone increasing $C^{1}$-function, viz

$$
N(\theta)>0, \quad F_{2}^{\prime}(\chi)>0 \quad \forall(\theta, \chi) .
$$

In connection with (2.13), we finally achieve

$$
\frac{\partial \psi}{\partial \chi}(\theta, \chi)+N(\theta) F_{2}^{\prime}(\chi) \dot{\chi}=0
$$

Such an equation is a thermodynamically compatible constitutive relation governing the evolution of the phase variable $\chi$. It represents the general class of models we deal with. In the sequel, we are able to show that (2.14) summarizes a lot of wellknown phase transition models. In Section 3, moreover, special attention will be focused on some particular cases for the function $N(\theta)$.

\subsection{Expression of $B$ compatible with the phase transition behaviour}

As a consequence of (2.14), the evolution of $\chi$ is influenced by the dependence of the free energy on the phase variable. Now, allowing for the general expression (2.11) of $\psi$, we comment some peculiar choices of $B(\chi)$ by means of the stationary conditions. In essence, by paralleling the principle of virtual power under unilateral constraints, we can say that, for any fixed temperature $\theta, \chi_{e}$ is an admissible stationary value of the phase variable $\chi$ if and only if

$$
\frac{\partial \psi}{\partial \chi}\left(\theta, \chi_{e}\right) \delta \chi \geq 0
$$

for every virtual phase variation $\delta \chi$ compatible with the domain of $\chi$, namely $[0,1]$. In other words, we have that

$$
\left.\frac{\partial \psi}{\partial \chi}\left(\theta, \chi_{e}\right)=0 \quad \text { for internal equilibrium points } \chi_{e} \in\right] 0,1[
$$

and

$$
\left.\frac{\partial \psi}{\partial \chi}\right|_{\chi_{e}=0} \geq 0 \quad \text { or }\left.\quad \frac{\partial \psi}{\partial \chi}\right|_{\chi_{e}=1} \leq 0
$$

for equilibrium at the end points. In addition, by standard requirements about stability of equilibria, the free energy $\psi$ is demanded to take its minimum values in such stationary points. Thus, the stationary behaviour of the material at hand will constrain the admissible expression of the function $B(\chi)$.

(i) First, we discuss the choice of $B(\chi)$ in connection with the existence of a "mushy region" at the equilibrium critical temperature $\theta=\theta_{c}$. At such a fixed value, free energy depends on $\chi$ through $B$, only, and takes the form

$$
\psi\left(\theta_{c}, \chi\right)=-\theta_{c} F_{0}\left(\theta_{c}\right)+\theta_{c} B(\chi) .
$$


We distinguish two possible occurrences.

$\left(i_{a}\right)$ If a "mushy region" does exist at $\theta=\theta_{c}$, then all possible values $\chi_{e} \in[0,1]$ are allowed at equilibrium. In this case, function $B$ must be convex and assume a constant value on $[0,1]$. Actually, the indicator function $I$ of the interval $[0,1]$, i.e.,

$$
I(\chi)= \begin{cases}0 & \text { if } \chi \in[0,1] \\ +\infty & \text { otherwise }\end{cases}
$$

satisfies such a property and, further, it forces the phase variable $\chi$ to sit between 0 and 1 . Thus, the choice $B=I$ is well justified. In this case, $B$ has no classical derivatives in $\mathbb{R}$, but, since $I$ is a proper convex lower semicontinuous function, its subdifferential $\partial I$ exactly meets our purposes. Indeed, note that $\partial I$ coincides with the inverse $H^{-1}$ of the Heaviside graph $H$ (specified by $H(\xi)=0$ if $\xi<0, H(0)=[0,1], H(\xi)=1$ if $\xi>0$ ) and

$$
\zeta_{e} \in \partial I\left(\chi_{e}\right) \quad \text { if and only if } \zeta_{e}\left(\chi_{e}-\xi\right) \leq 0 \quad \forall \xi \in[0,1] \text {. }
$$

$\left(i_{b}\right)$ According to material properties of the phase change, the "mushy region" may be physically unmotivated. If so, the only admissible equilibrium values for the phase variable are $\chi=0$ and $\chi=1$. In such a case, function $B(\chi)$ must exhibit two strict minima at $\chi=0$ and $\chi=1$ and the convexity property fails to hold. Usually $B$ is represented by a double well function.

(ii) Now, taking into account temperature values far from the critical one, and reminding that $F_{1}^{\prime}(\chi) \geq 0$, we examine the admissible choices for $B$ in connection with the existence of superheating and undercooling effects under stationary conditions. b" Indeed, if $F_{1} \neq 0$, any model related to $\left(i_{a}\right)$ exhibits supercooling and underheating effects under kinetic conditions (see, e.g., Refs. 21, 22). In what follows, for simplicity, we consider the symmetric case, when both effects occur or none of them.

(ii $i_{a}$ Superheating and undercooling effects do not occur under stationary conditions so that, for any temperature, $\psi$ exhibits only one minimum with respect to $\chi$ which is necessarily located at $\chi=0$ for $\theta<\theta_{c}$, and at $\chi=1$ for $\theta>\theta_{c}$. As a consequence, at critical temperature $\theta=\theta_{c}$, both $\chi=0$ and $\chi=1$ are allowed to minimize free energy. Since $F_{1}$ is required to be a monotone increasing function in $[0,1]$, it is quite reasonable to let $B=I$.

$\left(i i_{b}\right)$ Both superheating and undercooling effects occur. In such a case, $\psi$ must exhibit two strict minima at $\chi=0$ and $\chi=1$ for all temperatures $\theta$ such that $\left|\theta-\theta_{c}\right| / \theta_{c}$ is sufficiently small, in particular when $\theta=\theta_{c}$. We are thus compelled to assume that $B(\chi)$ is a non-convex double-well function. As $F_{1}$ is nondecreasing, $\psi$ takes its absolute minimum at $\chi=1$ when $\theta>\theta_{c}$, and at $\chi=0$ when $\theta<\theta_{c}$.

As an example of $\left(i i_{b}\right)$, let consider

$$
B(\chi)=\nu \chi^{2}(\chi-1)^{2} \quad, \quad F_{1}(\chi)=\frac{\lambda}{3} \chi^{2}(3-2 \chi)
$$


with $\lambda>\nu>0$. Letting $u(\theta)=\lambda\left(\theta-\theta_{c}\right) /(\nu \theta)$, from (2.11) and (2.15) one easily obtains

$$
\frac{\partial \psi}{\partial \chi}=4 \nu \theta \chi(\chi-1)\left(\chi-\frac{1-u(\theta)}{2}\right) .
$$

If $u(\theta) \in]-1,1[$, then the free energy takes two minima, at $\chi=0$ and $\chi=1$, so that the superheating and undercooling phenomena occur when $\theta$ lies in the temperature region

$$
\left\{\frac{\lambda \theta_{c}}{\lambda+\nu}<\theta<\frac{\lambda \theta_{c}}{\lambda-\nu}\right\} .
$$

Incidentally, the functions $B$ and $F_{1}$ of example (2.15) are quite similar to those considered by Wheeler and McFadden ${ }^{24}$ in discussing a modified Allen-Cahn model. ${ }^{1}$ We make a comparison with such a model in the next subsection.

Remark 2.1. The situations depicted by $\left(i_{a}\right)$ and $\left(i i_{a}\right)$ are strictly related, in the sense that both postulate the position $B=I$. On the other hand, chances $\left(i_{b}\right)$ and $\left(i i_{b}\right)$ are only compatible with functions $B$ possessing two distinct minima. Therefore, in the rigid phase transitions described by (2.11), if a mushy region exists at $\theta=\theta_{c}$, then undercooling and superheating effects do not occur at equilibrium, and viceversa.

In order to avoid $\chi$ to assume values outside the unitary interval $[0,1]$, but preserving strict minima at the ends, the indicator function may be added to either a double-well or a concave function (the final result will show a double well anyhow in cases $\left(i_{b}\right)$ and $\left(i i_{b}\right)$, due to the behaviour of the indicator function $I$ outside $\left.[0,1]\right)$. Therefore, we are led to assume henceforth

$$
B(\chi)=I(\chi)+B_{1}(\chi)
$$

where $B_{1}$ stands for a smooth function forcing $B$ to have only two minima at $\chi=0$ and $\chi=1$. On the other hand, $B_{1}$ may be taken as the null function in chances $\left(i_{a}\right)$ and $\left(i i_{a}\right)$.

\subsection{Comparison with previous models}

In this section we show that some previously stated models can be viewed as particular cases of our general setting, which is summarized by assumptions (2.11), viz

$$
\psi(\theta, \chi)=-\theta F_{0}(\theta)+\theta B(\chi)-\left(\theta-\theta_{c}\right) F_{1}(\chi),
$$

and (2.14), that is

$$
\frac{\partial \psi}{\partial \chi}+N(\theta) F_{2}^{\prime}(\chi) \dot{\chi}=0,
$$

where $B=I+B_{1}$.

\subsubsection{Finite-memory models}

Such a class of models physically exhibits a finite-memory relation between the phase variable $\chi$ and the temperature $\theta$. More precisely, if we define, for each fixed 
time $t>0, \bar{t}_{0}=\sup \left\{\bar{t} \in\left[0, t\left[: \theta(\bar{t})=\theta_{c}\right\}\right.\right.$ or $\bar{t}_{0}=0$ if the set $\left\{\bar{t} \in\left[0, t\left[: \theta(\bar{t})=\theta_{c}\right\}\right.\right.$ is empty, setting $\chi_{0}=\chi\left(\bar{t}_{0}\right)$ and

$$
\tilde{\theta}(t)=\int_{\bar{t}_{0}}^{t} \frac{\theta(\tau)-\theta_{c}}{N(\theta(\tau))} d \tau
$$

a finite-memory model can be characterized by a suitable relationship between $\chi$ and $\tilde{\theta}$. In particular, we restrict our attention to the following

$$
\chi= \begin{cases}0 & \text { if } G\left(\tilde{\theta}+G^{-1}\left(\chi_{0}\right)\right) \leq 0 \\ 1 & \text { if } G\left(\tilde{\theta}+G^{-1}\left(\chi_{0}\right)\right) \geq 1 \\ G\left(\tilde{\theta}+G^{-1}\left(\chi_{0}\right)\right) & \text { otherwise }\end{cases}
$$

where $G$ is an odd and increasing $C^{1}$-function, whose expression depends on the nature of the material. If we sketch this model in the $(\tilde{\theta}, \chi)$-plane, we observe that the representative point $A$ describes a cycle, quite similar to a hysteresis loop, as the temperature varies continuously around $\theta_{c}$. Actually, suppose to start at time $t_{0}$ from the origin $(0,0)$ of the plane and let the relative temperature be positive, i.e., $\theta>\theta_{c}$. As a consequence, $\left(\theta-\theta_{c}\right) / N(\theta)$ is a positive function of time, so that $\tilde{\theta}$ increases. It is worth noting that $\tilde{\theta}$ keeps on increasing provided that temperature stays beyond its critical value. At some point $\tilde{\theta}=\tilde{\theta}_{0}, \chi$ reaches its maximum (namely, $\chi=1$ ) and keeps it as long as $\theta>\theta_{c}$. If $\theta-\theta_{c}$ vanishes at some instant $t=t_{1}$ and becomes negative afterwards, then $\bar{t}_{0}$ changes its value into $t_{1}$. According to $(2.16)$, the point $A$ suddenly jumps to $(0,1)$, then $\chi$ decreases until it reaches its minimum (that is, $\chi=0$ ). The nonlinearity $G$ contributes to the above graph by fixing the shape of the curves described by $A$. In particular, the values of $\tilde{\theta}_{0}$ is related to $G$ through $\tilde{\theta}_{0}=G^{-1}(1)-G^{-1}\left(\chi_{0}\right)$.

Constitutive relation (2.16) is quite new and exhibits loops like those involved in hysteretic phenomena. When $N(\theta)=1$, such a model is similar to those proposed by Fabrizio and Gentili. ${ }^{12}$ However, as we are going to show, here the pair $(\theta, \chi)$ is sufficient to describe the state of a material governed by (2.16), whereas the general models of Fabrizio and Gentili need the additional state variable $\tilde{\theta}$.

Now we prove that (2.16) is provided by our general setting when we assume $B_{1}=0$ and $F_{1}^{\prime}>0$. In fact, in this case (2.14) takes the form

$$
\theta H^{-1}(\chi) \ni\left(\theta-\theta_{c}\right) F_{1}^{\prime}(\chi)-N(\theta) F_{2}^{\prime}(\chi) \dot{\chi} .
$$

In order to compare (2.16) with (2.17), let $J=G^{-1}$. Because of the strict monotonicity assumed for $G$ and $J,(2.16)$ can be rewritten as

$$
J(\chi)= \begin{cases}0 & \text { if } \tilde{\theta}+J\left(\chi_{0}\right) \leq 0 \\ J(1) & \text { if } \tilde{\theta}+J\left(\chi_{0}\right) \geq J(1) \\ \tilde{\theta}+J\left(\chi_{0}\right) & \text { otherwise }\end{cases}
$$


Consider now two instants $t_{0,1}$ and $t_{0,2}>t_{0,1}$ such that

$$
\left.\theta\left(t_{0,1}\right)=\theta\left(t_{0,2}\right)=\theta_{c} \quad, \quad \theta(t) \neq \theta_{c} \quad \text { for } t \in\right] t_{0,1}, t_{0,2}[
$$

and set $\chi_{0,1}=\chi\left(t_{0,1}\right)$ and $\chi_{0,2}=\chi\left(t_{0,2}\right)$. It is not difficult to check that

$$
\lim _{t \rightarrow t_{0,2}^{-}} \frac{\partial}{\partial t}\left(\tilde{\theta}+J\left(\chi_{0,1}\right)\right)=\lim _{t \rightarrow t_{0,2}^{+}} \frac{\partial}{\partial t}\left(\tilde{\theta}+J\left(\chi_{0,2}\right)\right)=0
$$

Therefore, we can continuously differentiate (2.18), so yielding

$$
J^{\prime}(\chi) \dot{\chi}=\left\{\begin{array}{ll}
\left(\theta-\theta_{c}\right) / N(\theta) & \text { if } \left.\tilde{\theta}+J\left(\chi_{0}\right)=J(\chi) \in\right] 0, J(1)[ \\
0 & \text { otherwise }
\end{array} .\right.
$$

Since $J$ is increasing in $[0,1]$ and $J(0)=0,(2.19)$ implies

$$
\begin{aligned}
& \left.\frac{\theta-\theta_{c}}{N(\theta)}-J^{\prime}(\chi) \dot{\chi}=0 \quad \text { for } \quad \chi \in\right] 0,1[ \\
& \theta-\theta_{c} \geq 0, J^{\prime}(\chi) \dot{\chi}=0 \quad \text { for } \chi=1, \\
& \theta-\theta_{c} \leq 0, J^{\prime}(\chi) \dot{\chi}=0 \quad \text { for } \chi=0
\end{aligned}
$$

and can be summarized as follows

$$
\frac{\theta-\theta_{c}}{N(\theta)}-J^{\prime}(\chi) \dot{\chi} \in H^{-1}(\chi)
$$

Therefore, (2.20) states the "rate type" constitutive relation (inclusion) corresponding to (2.16) and involving $\chi$ and $\theta$ only. Taking into account that positive factors can be omitted in front of $H^{-1},(2.17)$ is easily seen to be equivalent to $(2.20)$ provided

$$
F_{1}^{\prime}(\chi)>0 \text { for all } \chi \in[0,1] \text { and } \quad J^{\prime}(\chi)=\frac{F_{2}^{\prime}(\chi)}{F_{1}^{\prime}(\chi)}
$$

\subsubsection{Stefan problem and relaxed Stefan problem}

The relaxed Stefan problem (see Refs. 20-23) can be derived from our framework by assuming $B_{1}(\chi)=0$ and $F_{1}(\chi)=L \chi / \theta_{c}$, namely

$$
\psi(\theta, \chi)=-\theta F_{0}(\theta)+\theta I(\chi)-\frac{L}{\theta_{c}}\left(\theta-\theta_{c}\right) \chi
$$

In this case, the constitutive equation for $\chi$ becomes

$$
\frac{L\left(\theta-\theta_{c}\right)}{\theta_{c} N(\theta)} \in H^{-1}(\chi)+F_{2}^{\prime}(\chi) \dot{\chi}
$$

in view of $\partial I=H^{-1}$. Then, the phase relaxation system studied by Visintin ${ }^{20-22}$ is obtained when $F_{2}^{\prime}$ is a (positive) constant. 
On the other hand, a linearization of the internal energy expression (cf. (2.3) and (2.8)), namely

$$
e=c_{0} \theta+L \chi
$$

corresponds to the choice $F_{0}^{\prime}(\theta)=c_{0} / \theta$. In such a case, the related free energy

$$
\psi(\theta, \chi)=-c_{0} \theta \ln \theta+\theta I(\chi)-\frac{L}{\theta_{c}}\left(\theta-\theta_{c}\right) \chi
$$

is basically the same as in Frémond and Visintin. ${ }^{15}$ Incidentally, the dissipation pseudopotentials used there are of type

$$
U(\theta, \chi, \dot{\chi})=\frac{1}{2} g(\chi, \theta) \dot{\chi}^{2}
$$

which can be combined with (2.13). The classical Stefan problem may be regarded as the limiting case as $F_{2}^{\prime}$ goes to 0 , whence the constitutive equation for $\chi$ is

$$
\left(\theta-\theta_{c}\right) \in H^{-1}(\chi) \quad \text { or, equivalently, } \quad \chi \in H\left(\theta-\theta_{c}\right)
$$

We conclude this subsection by commenting on two phase field models. Actually, such models are not completely included in our situation since they are based on the assumption that $\chi$ is either 0 or 1 in the most part of the body and the two phases are separated by a narrow diffusive interface. Therefore, the expression of the free energy density also contains a term $\varepsilon|\nabla \chi|^{2}$, representing the interfacial energy, and the indicator function $I(\chi)$ may be replaced by other convex functions (cf., e.g., (2.15)), but with the sum $B$ having two strict minima in 0 and 1 . Nevertheless, we can compare such models with our setting, by considering their limiting case when the interfacial energy term vanishes (that is, $\varepsilon \searrow 0$ ).

\subsubsection{Standard phase field model}

The standard phase field model is mainly due to Cahn ${ }^{1,7}$ and coworkers, and it has been subsequently analised by Caginalp. ${ }^{6}$ In order to carry out some comparison, it is worth considering the modified Allen-Cahn free energy $\psi^{*}$ due to Wheeler and McFadden ${ }^{24}$ and mentioned above. Indeed, apart from the interfacial energy term, $\psi^{*}$ exhibits the form

$$
\psi^{*}(\theta, \chi)=-\theta F_{0}(\theta)+\frac{1}{4 a} \chi^{2}(1-\chi)^{2}-\frac{L\left(\theta-\theta_{c}\right)}{\theta_{c}} \chi^{2}(3-2 \chi)
$$

where $L$ still denotes the latent heat, and $1 / a$ is proportional to the barrier height of the double-well potential. Even if $a$ is often taken as a constant, we note that this gap usually depends on temperature (see Ref. 24, p. 371). In particular, if we put

$$
\frac{1}{a}=4 \nu \theta \quad \text { and } \quad L=\frac{1}{3} \lambda \theta_{c}
$$


then $(2.22)$ is equivalent to $(2.11),(2.15)$. Such a choice leads to a free energy which exhibits a double well depending on temperature. Moreover, the related internal energy $e$, given by (2.3), is monotone increasing with respect to both $\theta$ and $\chi$. On the contrary, when $1 / a$ is assumed to be constant in (2.22), owing to (2.8) the related internal energy $e^{*}$ takes the form

$$
e^{*}(\theta, \chi)=\theta^{2} F_{0}^{\prime}(\theta)+\frac{1}{4 a} \chi^{2}(1-\chi)^{2}+L \chi^{2}(3-2 \chi)
$$

and is non-monotone with respect to $\chi$. Such a situation has been scrutinized by Caginalp ${ }^{6}$.

\subsubsection{Penrose-Fife model}

In recent years Penrose and Fife developed a phase field model on the basis of thermodynamic arguments, assuming the following expression for the internal energy

$$
e(\theta, \chi)=c(\chi) f_{0}(\theta)+f_{1}(\chi)
$$

If no interfacial energy is present and $c$ is a constant function, then the resulting phase-field relationship is

$$
\mu \dot{\chi}+\partial I(\chi) \ni s^{\prime}(\chi)-\frac{f_{1}^{\prime}(\chi)}{\theta} .
$$

Thus, taking $B_{1} \neq 0, N(\theta)=\theta, F_{2}(\chi)=\mu \chi$, it is easy to check that (2.14) becomes

$$
\mu \dot{\chi}+\partial I(\chi) \ni B_{1}^{\prime}(\chi)+\frac{\theta-\theta_{c}}{\theta} F_{1}^{\prime}(\chi)
$$

which is equivalent to $(2.23)$ if we put

$$
s(\chi)=F_{1}(\chi)+B_{1}(\chi) \quad \text { and } \quad f_{1}(\chi)=\theta_{c} F_{1}(\chi)
$$

Therefore the non-diffusive Penrose-Fife equation can also be derived from our setting by means of suitable choices of the constitutive functions.

\section{Analysis of a particular model}

In this section we focus on the initial boundary value problem for the system of equations $(2.3),(2.4),(2.6),(2.11),(2.14)$. Therefore, the balance of energy in (2.6) leads to

$$
\frac{\partial}{\partial t}\left(\theta^{2} F_{0}^{\prime}(\theta)+\theta_{c} F_{1}(\chi)\right)-\operatorname{div}(\kappa(\theta) \nabla \theta)=r
$$

while, in the case when $B=I+B_{1},(2.11)$ and (2.14) yield

$$
\frac{\partial}{\partial t} F_{2}(\chi)+\partial I(\chi)+\frac{\theta}{N(\theta)} B_{1}^{\prime}(\chi) \ni \frac{\theta-\theta_{c}}{N(\theta)} F_{1}^{\prime}(\chi)
$$


We complement this evolution problem with adiabatic boundary conditions

$$
\mathbf{q} \cdot \mathbf{n}=-\kappa(\theta) \frac{\partial \theta}{\partial \mathbf{n}}=0 \quad \text { on } \Sigma=\partial \Omega \times(0, T),
$$

and the initial conditions (obviously given for the internal energy and the pseudopotential $\left.F_{2}(\chi)\right)$

$$
\left.\left(\theta^{2} F_{0}^{\prime}(\theta)+\theta_{c} F_{1}(\chi)\right)\right|_{t=0}=e_{0} \quad,\left.\quad\left(F_{2}(\chi)\right)\right|_{t=0}=\varphi_{0} .
$$

Let us notice that $\mathbf{n}$ represents the outward normal unit vector to $\partial \Omega$ and $\partial / \partial \mathbf{n}$ stands for the related normal derivative.

We recall that several models with non-convex $B$ have recently received a good deal of attention in the literature. In particular, the reduced Penrose-Fife system, resulting from the choices $B_{1} \neq 0$ and $N(\theta)=\theta$, seems to be of some interest. In such a case, dividing (3.1) by $F_{2}^{\prime}(\chi)>0$, the consequent inclusion reads

$$
\dot{\chi}+\partial I(\chi)+\frac{B_{1}^{\prime}(\chi)}{F_{2}^{\prime}(\chi)} \ni \frac{\theta-\theta_{c}}{\theta} \frac{F_{1}^{\prime}(\chi)}{F_{2}^{\prime}(\chi)} .
$$

Related initial boundary value problems have been studied by Colli and Sprekels ${ }^{9,10}$ under two different constitutive laws for $\kappa(\theta)$. In Ref. 9 (see also Ref. 19), the heat flux is supposed to be proportional to the gradient of the inverse of the absolute temperature, namely $\kappa(\theta)=-k / \theta^{2}$, while the case, more delicate from the mathematical point of view, of a linear Fourier law (i.e., (2.4) with $\kappa(\theta)=k$ positive constant), is treated in Ref. 10.

In the sequel, we analyse the situation in which $\kappa(\theta)=k$ and $N(\theta)$ is a given Lipschitz continuous function, bounded from below by a positive constant (for instance, one may have $N(\theta)=\theta_{*}$, for some fixed temperature $\theta_{*}$, or $N(\theta)=\max \left\{\theta, \theta_{*}\right\}$ ). Thus, the ensuing model differs from the Penrose-Fife and standard phase-field models. Moreover, by looking at other phase relaxation systems (cf. especially Refs. 2, $3,8,11,15,20-22)$, it turns out that earlier existence results do not apply to our initial boundary value problem and, as far as we know, the maximum principle property yielding positivity of temperature (see Subsection 3.4) was not previously investigated. We conclude this premise by stressing the physical admissibility of the above fixed system, on account of the thermodynamically consistent family of models outlined in the previous section.

\subsection{Main result}

Let $\Omega \subseteq \mathbb{R}^{3}$ be a bounded domain with smooth boundary $\partial \Omega$. For a given final time $T>0$, we fix the cylinder $Q=\Omega \times(0, T)$ and its lateral boundary $\Sigma=\partial \Omega \times(0, T)$. Our problem consists in finding a pair of functions, $\theta: Q \rightarrow \mathbb{R}$ and $\chi: Q \rightarrow[0,1]$, regular enough and such that the relations

$$
\begin{aligned}
& \frac{\partial}{\partial t}\left(\theta^{2} F_{0}^{\prime}(\theta)+\theta_{c} F_{1}(\chi)\right)-k \Delta \theta=0 \\
& \frac{\partial}{\partial t} F_{2}(\chi)+\partial I(\chi)+\frac{\theta B_{1}^{\prime}(\chi)}{N(\theta)} \ni \frac{\theta-\theta_{c}}{N(\theta)} F_{1}^{\prime}(\chi)
\end{aligned}
$$


are satisfied a.e. in $Q$, as well as

$$
\frac{\partial \theta}{\partial \mathbf{n}}=0 \quad \text { a.e. in } \Sigma
$$

and

$$
\left(\theta^{2} F_{0}^{\prime}(\theta)+\theta_{c} F_{1}(\chi)\right)(\mathbf{x}, 0)=e_{0}(\mathbf{x}), \quad F_{2}(\chi)(\mathbf{x}, 0)=\varphi_{0}(\mathbf{x}) \quad \text { for a.a. } \mathbf{x} \in \Omega .
$$

Clearly, we are interested to (3.2-5) for suitable choices of the data, which must be in accord with the physical framework. First of all, we assume that the specific heat $c_{v}=(\partial e / \partial \theta)\left(=2 \theta F_{0}^{\prime}(\theta)+\theta^{2} F_{0}^{\prime \prime}(\theta)\right)$ is well defined and lies between two positive constants $C_{*}$ and $C^{*}$. This is fulfilled whenever $F_{0}^{\prime}$ is a.e. differentiable and

$$
C_{*} \leq \zeta\left(2 F_{0}^{\prime}(\zeta)+\zeta F_{0}^{\prime \prime}(\zeta)\right) \leq C^{*} \quad \text { for a.a. } \zeta \in \mathbb{R} \text {. }
$$

Of course, $F_{0}$ should be defined only in the interval ]0, $+\infty\left[\right.$, but we let $\zeta \mapsto \zeta^{2} F_{0}^{\prime}(\zeta)$ work on the entire $\mathbb{R}$ and refer to the later Remark 3.7. Moreover, we suppose

$$
N \in C^{0,1}(\mathbb{R}), \quad N(\zeta) \geq \theta_{*}>0 \quad \forall \zeta \in \mathbb{R}
$$

and let $F_{1}, F_{2}$ and $B_{1}$ be smooth functions with $F_{2}$ satisfying the monotonicity property mentioned in Section 2, namely,

$$
\begin{gathered}
F_{1}, F_{2}, B_{1} \in C^{2}([0,1]), \\
F_{2}^{\prime}(\xi)>0 \quad \forall \xi \in[0,1], \quad \frac{1}{F_{2}^{\prime}} \in L^{\infty}(0,1) .
\end{gathered}
$$

Finally, we prescribe $\theta_{0}, \chi_{0} \in L^{2}(\Omega)$ with

$$
\theta_{0} \in H^{1}(\Omega), \quad 0 \leq \chi_{0} \leq 1 \quad \text { a.e. in } \Omega,
$$

so that initial data in (3.5) are given by

$$
e_{0}=\theta_{0}^{2} F_{0}^{\prime}\left(\theta_{0}\right)+\theta_{c} F_{1}\left(\chi_{0}\right), \quad \varphi_{0}=F_{2}\left(\chi_{0}\right) \text {. }
$$

On the other hand, one can alternatively assign $e_{0}$ and $\varphi_{0}$, with $F_{2}(0) \leq \varphi_{0} \leq F_{2}(1)$, and then recover $\chi_{0}=F_{2}^{-1}\left(\varphi_{0}\right)$ and $\theta_{0}$ via the above equalities. Concerning the assumptions on $F_{1}, F_{2}, B_{1}$, we point out that in our analysis we do not need any monotonicity property for the function $F_{1}$ (as it was instead postulated in $(2.1-$ $3)$ ). Therefore, our results actually apply to even more general situations like, for instance, models for solid-solid phase transitions (see, e.g., the free energy expression coming out from the position (1.8) in Ref. 10).

Next, we set problem (3.2-5) in a convenient formulation. First, we fix a notation for the function in (3.6), letting

$$
\alpha(\zeta):=\zeta\left(2 F_{0}^{\prime}(\zeta)+\zeta F_{0}^{\prime \prime}(\zeta)\right), \quad \zeta \in \mathbb{R}
$$


so that

$$
\alpha \in L^{\infty}(\mathbb{R}), \quad C_{*} \leq \alpha(\zeta) \leq C^{*} \quad \text { for a.a. } \zeta \in \mathbb{R} .
$$

Then, for the sake of simplicity, we also introduce

$$
\beta_{1}(\xi):=\frac{F_{1}^{\prime}(\xi)-B_{1}^{\prime}(\xi)}{F_{2}^{\prime}(\xi)}, \quad \beta_{2}(\xi):=\frac{F_{1}^{\prime}(\xi)}{F_{2}^{\prime}(\xi)}, \quad \xi \in[0,1]
$$

whence

$$
\beta_{1}, \beta_{2} \in C^{1}([0,1]), \quad \beta_{2}(\xi) F_{1}^{\prime}(\xi) \geq 0 \quad \forall \xi \in[0,1]
$$

because of (3.8-9). Observe that the product $\beta_{1} F_{1}^{\prime}$ has no sign property (contrary to $\beta_{2} F_{1}^{\prime}$ ) owing to the derivative of the function $B_{1}$.

Now, one sees that equation (3.3) can be slightly modified by dividing all terms by $F_{2}^{\prime}(\chi)>0$. Remarking that this division does not change the subdifferential $\partial I=H^{-1}$ and rewriting the inclusion as a variational inequality (cf. point $\left(i_{a}\right)$ in Subsection 2.2), we come to an equivalent formulation of the problem (3.2-5). In what follows, we also prescribe the regularity that is required for the solutions. In particular, we seek strong solutions (according to the usual terminology) as we want that equations and conditions are satisfied almost everywhere.

Problem (P). Find the pair $(\theta, \chi)$ satisfying

$$
\begin{gathered}
\theta \in H^{1}\left(0, T ; L^{2}(\Omega)\right) \cap C^{0}\left([0, T] ; H^{1}(\Omega)\right) \cap L^{2}\left(0, T ; H^{2}(\Omega)\right), \\
\chi \in H^{1}\left(0, T ; L^{2}(\Omega)\right), \\
\alpha(\theta) \dot{\theta}+\theta_{c} F_{1}^{\prime}(\chi) \dot{\chi}-k \Delta \theta=0 \quad \text { a.e. in } Q, \\
0 \leq \chi \leq 1 \quad \text { a.e. in } Q, \\
\dot{\chi}(\chi-\xi) \leq \frac{1}{N(\theta)}\left(\theta \beta_{1}(\chi)-\theta_{c} \beta_{2}(\chi)\right)(\chi-\xi) \quad \forall \xi \in[0,1], \text { a.e. in } Q, \\
\frac{\partial \theta}{\partial \mathbf{n}}=0 \quad \text { a.e. in } \Sigma, \\
\theta(\cdot, 0)=\theta_{0}, \quad \chi(\cdot, 0)=\chi_{0} \quad \text { a.e. in } \Omega .
\end{gathered}
$$

We point out that, by virtue of (3.13-14), the terms $\alpha(\theta) \dot{\theta}+\theta_{c} F_{1}^{\prime}(\chi) \dot{\chi}$ and $\dot{\chi}$ in (3.15) and (3.17) actually coincide with the terms $\left(\partial\left(\theta^{2} F_{0}^{\prime}(\theta)+\theta_{c} F_{1}(\chi)\right) / \partial t\right)$ and $\left(\partial F_{2}(\chi) / \partial t\right) / F_{2}^{\prime}(\chi)$ of $(3.2-3)$. For the above problem, we prove not only existence and uniqueness, but some regularity including the expected and reasonable property that the variable $\theta$, standing for the absolute temperature, attains non-negative values only.

Theorem 3.1. Problem $(P)$ admits a unique solution $(\theta, \chi)$.

The proof of such result is given in Subsections 3.2 and 3.3.

Remark 3.2. The presence of a nonlinear heat supply $r(\mathbf{x}, t, \theta, \chi)$ in the right hand side of (3.15) (and of (3.2)) would not affect the conclusion of this theorem 
provided $r(\mathbf{x}, t, \zeta, \xi)$ is measurable in $(\mathbf{x}, t)$ for any $(\zeta, \xi) \in \mathbb{R} \times[0,1]$ and Lipschitz continuous in $(\zeta, \xi)$ uniformly with respect to $(\mathbf{x}, t) \in Q$. The reader can directly check such a statement by following and extending our arguments.

Remark 3.3. A basic and useful reference for our existence proof is provided by Colli and Hoffmann, ${ }^{8}$ where a general theory is developed for multi-component phase relaxation systems. However the abstract existence result of Ref. 8 does not cover our case, since the Lipschitz continuity demanded for the nonlinearity of (3.17) fails because of the term $\theta \beta_{1}(\chi) / N(\theta)$. Moreover, no boundedness or positivity of temperature is discussed there. On the contrary, here the following theorems establish such properties provided the data are physically admissible.

Theorem 3.4. Assume $\theta_{0} \in L^{\infty}(\Omega)$ besides (3.10) for the initial data. Then the solution $(\theta, \chi)$ of $(P)$ satisfies

$$
\theta, \dot{\chi} \in L^{\infty}(Q)
$$

Theorem 3.5. Let the initial (absolute) temperature $\theta_{0}$ be non-negative almost everywhere in $\Omega$. Then the solution $(\theta, \chi)$ to $(P)$ is such that

$$
\theta \geq 0 \quad \text { a.e. in } Q \text {. }
$$

Both proofs are postponed till Subsection 3.4.

Remark 3.6. Referring to Remark 3.2, we point out that also in this case, by adapting the arguments outlined in the proofs, it is easy to check that (3.20-21) still hold in presence of a heat source $r$ whenever $r(\cdot, \cdot, 0,0) \in L^{\infty}(Q)$, for instance, and

$$
r(\cdot, \cdot, \zeta, \xi) \geq 0 \quad \text { a.e. in } Q, \text { for all } \zeta<0 \text { and } \xi \in[0,1]
$$

in addition to the properties listed in Remark 3.2. It is worth noting that the last requirement is not just suggested by mathematical convenience. On the contrary, it is based on the reasonable physical assumption that the internal energy reaches its minimum value at the zero absolute temperature. As a consequence, whatever physically admissible heat source must be non-negative at such a temperature and, what is more, at (physically not allowable) negative temperatures.

Remark 3.7. Concerning Theorem 3.4, note that (3.16) and (3.20) yield $\chi \in$ $W^{1, \infty}(Q)$. Moreover, if one collects the conclusions of both theorems, it turns out that there exists some positive value $\Theta\left(\geq \theta_{0}\right)$ such that $0 \leq \theta \leq \Theta$ almost everywhere in $Q$. Owing to this fact, we observe that for our aims it would be essential to know the function $\zeta \mapsto \zeta^{2} F_{0}^{\prime}(\zeta)$ (as well as $N$, see (3.6-7)) only in the interval $[0, \Theta]$, as this function can be easily extended to the entire $\mathbb{R}$ keeping the validity 
of (3.6) (the same could be said for the dependence of $r$ on $\theta$, if any right hand side $r(\mathbf{x}, t, \theta, \chi)$ is added to (3.15)).

The collection of the results stated in this subsection can be compared with Theorem 2.2 in Ref. 10, which basically refers to the problem (3.15-19) resulting from the choice $N(\theta)=\theta$. There, under suitable assumptions on the data, it is shown that $\theta$ remains bounded from below by a positive constant (so that $1 / N(\theta)$ is well defined and lies in $L^{\infty}(Q)$ ). Here, it is just proved that $\theta$ is non-negative, but we do not need any compatibility conditions between $B_{1}^{\prime}$ and $F_{1}^{\prime}$ (as it was instead in hypothesis (A4) of Ref. 10).

\subsection{Uniqueness}

In order to show Theorem 3.1, first we check that Problem (P) has at most one solution. Arguing by contradiction, let the pairs $\left(\theta_{1}, \chi_{1}\right)$ and $\left(\theta_{2}, \chi_{2}\right)$ both solve $(\mathrm{P})$ and put

$$
\theta=\theta_{1}-\theta_{2} \quad, \quad \chi=\chi_{1}-\chi_{2} .
$$

Integrating the difference of the two equations (3.15) with respect to time, as $\theta(\cdot, 0)=\chi(\cdot, 0)=0$ a.e. in $\Omega$, we obtain

$$
\begin{gathered}
\left(A\left(\theta_{1}\right)-A\left(\theta_{2}\right)+\theta_{c}\left(F_{1}\left(\chi_{1}\right)-F\left(\chi_{2}\right)\right)\right)(\mathbf{x}, t)-k \Delta \int_{0}^{t} \theta(\mathbf{x}, s) d s=0 \\
\text { for a.a. }(\mathbf{x}, t) \in Q,
\end{gathered}
$$

where

$$
A(\zeta):=\int_{0}^{\zeta} \alpha(\xi) d \xi, \quad \zeta \in \mathbb{R}
$$

Then, multiply (3.22) by $\theta$ and integrate over $\left.\left.Q_{t}:=\Omega \times\right] 0, t[, t \in] 0, T\right]$, using (3.18). Owing to (3.11) and (3.9), it is straightforward to deduce

$$
C_{*} \iint_{Q_{t}}|\theta|^{2} d \mathbf{x} d s+\frac{k}{2}\left\|\nabla \int_{0}^{t} \theta(\cdot, s) d s\right\|^{2} \leq \theta_{c}\left\|F_{1}^{\prime}\right\|_{L^{\infty}(0,1)} \iint_{Q_{t}}|\theta \| \chi| d \mathbf{x} d s
$$

where $\|\cdot\|$ denotes the norm either in $L^{2}(\Omega)$ or $\left(L^{2}(\Omega)\right)^{3}$.

Next, we write (3.17) for $\chi_{1}$ choosing $\xi=\chi_{2}$ (and this is admissible thanks to (3.16)) and for $\chi_{2}$ taking $\xi=\chi_{1}$. In view of (3.7) and (3.12), the sum of the two inequalities and the subsequent integration plainly leads to

$$
\begin{aligned}
& \frac{1}{2}\|\chi(\cdot, t)\|^{2} \leq \frac{1}{\theta_{*}} \iint_{Q_{t}}\left(\left|\theta_{1}\right|\left\|\beta_{1}^{\prime}\right\|_{L^{\infty}(0,1)}+\left|\theta_{c}\right|\left\|\beta_{2}^{\prime}\right\|_{L^{\infty}(0,1)}\right)|\chi|^{2} d \mathbf{x} d s \\
& \quad+\left\|(1 / N)^{\prime}\right\|_{L^{\infty}(\mathbb{R})} \iint_{Q_{t}}\left(\left|\theta_{1}\right|\left\|\beta_{1}\right\|_{L^{\infty}(0,1)}+\left|\theta_{c}\right|\left\|\beta_{2}\right\|_{L^{\infty}(0,1)}\right)|\theta||\chi| d \mathbf{x} d s \\
& \quad+\frac{1}{\theta_{*}}\left\|\beta_{1}\right\|_{L^{\infty}(0,1)} \iint_{Q_{t}}|\theta||\chi| d \mathbf{x} d s .
\end{aligned}
$$


For the sake of simplicity, in the sequel we will often use the symbol $C$, with possible subscripts, to denote positive constants depending only on the data. Due to the dimension 3 of space, $H^{2}(\Omega)$ is continuously embedded in $L^{\infty}(\Omega)$. Hence, recalling (3.13), it turns out that

$$
\frac{1}{2}\|\chi(\cdot, t)\|^{2} \leq C \int_{0}^{t}\left(\left\|\theta_{1}(\cdot, s)\right\|_{H^{2}(\Omega)}+1\right)\|\chi(\cdot, s)\|(\|\chi(\cdot, s)\|+\|\theta(\cdot, s)\|) d s .
$$

Finally, combining (3.24) and (3.25), with the help of the elementary inequality

$$
a b \leq \frac{\varepsilon}{2} a^{2}+\frac{1}{2 \varepsilon} b^{2} \quad \forall a, b \in \mathbb{R}, \forall \varepsilon>0,
$$

we easily infer that

$$
\frac{C_{*}}{2} \iint_{Q_{t}}|\theta|^{2} d \mathbf{x} d s+\frac{1}{2}\|\chi(\cdot, t)\|^{2} \leq C \int_{0}^{t}\left(1+\left\|\theta_{1}(\cdot, s)\right\|_{H^{2}(\Omega)}^{2}\right)\|\chi(\cdot, s)\|^{2} d s
$$

for any $t \in[0, T]$. Since the function $t \mapsto 1+\left\|\theta_{1}(\cdot, t)\right\|_{H^{2}(\Omega)}^{2}$ belongs to $L^{1}(0, T)$ (because of (3.13)), the Gronwall lemma implies that the left hand side is zero for all $t \in[0, T]$, so that $\theta_{1}=\theta_{2}$ and $\chi_{1}=\chi_{2}$ a.e. in $Q$.

Remark 3.8. Note that the assumption $\Omega \subseteq \mathbb{R}^{3}$ has been already exploited to establish that $H^{2}(\Omega) \subset L^{\infty}(\Omega)$. Anyway, it may be worthwhile to point out that Theorem 3.1 holds true in dimensions 1 and 2 , as well.

\subsection{Existence}

The existence proof is based on an approximation method complemented with uniform estimates and passage to the limit, in which compactness and continuity arguments are used.

Our approximating scheme is chosen in a way to take advantage of the general results contained in Ref. 8. Then, for any $n \in \mathbb{I}$ we introduce the truncated identity

$$
\tau_{n}(\zeta)=\min \{\max \{\zeta,-n\}, n\}, \quad \zeta \in \mathbb{R},
$$

and replace $\theta$ with $\tau_{n}(\theta)$ as factor of $\beta_{1}(\chi)$ in (3.17).

Problem $\left(\mathbf{P}_{n}\right)$. Find the pair $\left(\theta_{n}, \chi_{n}\right)$ satisfing (3.13-16), (3.18-19) (where $\theta$ and $\chi$ must be substituted with $\theta_{n}$ and $\chi_{n}$, respectively) and such that

$$
\begin{array}{r}
\dot{\chi}_{n}\left(\chi_{n}-\xi\right) \leq \frac{1}{N\left(\theta_{n}\right)}\left(\tau_{n}\left(\theta_{n}\right) \beta_{1}\left(\chi_{n}\right)-\theta_{c} \beta_{2}\left(\chi_{n}\right)\right)\left(\chi_{n}-\xi\right) \\
\forall \xi \in[0,1], \text { a.e. in } Q .
\end{array}
$$

Owing to (3.7-8), (3.11-12) and to the boundedness and Lipschitz continuity of $\tau_{n}$, we can extract the following assertion from Theorem 1 of Ref. 8 .

Proposition 3.6. For any $n \in \mathbb{N}$ there exists a unique solution to Problem $\left(P_{n}\right)$.

Actually, the regularity $\theta_{n} \in L^{2}\left(0, T ; H^{2}(\Omega)\right)$ is not stated in Ref. 8, but a comparison in

$$
\alpha\left(\theta_{n}\right) \dot{\theta}_{n}-k \Delta \theta_{n}=-\theta_{c} F_{1}^{\prime}\left(\chi_{n}\right) \dot{\chi}_{n}
$$


(i.e., (3.15)) yields $\Delta \theta_{n} \in L^{2}\left(0, T ; L^{2}(\Omega)\right)$, which suffices to conclude by virtue of (3.18) and well-known elliptic estimates.

Now we aim to derive bounds, independent of $n$, for the pair $\left(\theta_{n}, \chi_{n}\right)$ with respect to suitable norms. First, consider the system composed by (3.27), (3.16) and by the initial condition for $\chi_{n}$ in (3.19). This is nothing but an evolution equation for a subdifferential operator, whence we can apply the abstract theory of Brézis. ${ }^{4}$ In particular, taking (3.7) and (3.10) into account, from Theorem 3.6, p. 37, of Ref. 4 we can conclude that

$$
\int_{0}^{t}\left|\dot{\chi}_{n}(\cdot, s)\right|^{2} d s \leq \frac{1}{\theta_{*}} \int_{0}^{t}\left|\tau_{n}\left(\theta_{n}\right) \beta_{1}\left(\chi_{n}\right)-\theta_{c} \beta_{2}\left(\chi_{n}\right)\right|^{2} d s \quad \forall t \in[0, T],
$$

almost everywhere in $\Omega$, for all $t \in[0, T]$. By integrating also in space, (3.12) enables us to infer that

$$
\iint_{Q_{t}}\left|\dot{\chi}_{n}\right|^{2} d \mathbf{x} d s \leq C \iint_{Q_{t}}\left(\left|\theta_{n}\right|^{2}+1\right) d \mathbf{x} d s
$$

Next, the formal test of (3.28) by $\dot{\theta}_{n}$ (rigorous computations are performed, for instance, in Ref. 8) gives

$C_{*} \iint_{Q_{t}}\left|\dot{\theta}_{n}\right|^{2} d \mathbf{x} d s+\frac{k}{2}\left\|\nabla \theta_{n}(\cdot, t)\right\|^{2} \leq \frac{k}{2}\left\|\nabla \theta_{0}\right\|^{2}+\theta_{c}\left\|F_{1}^{\prime}\right\|_{L^{\infty}(0,1)} \iint_{Q_{t}}\left|\dot{\chi}_{n}\right|\left|\dot{\theta}_{n}\right| d \mathbf{x} d s$

thanks to (3.11) and (3.19). Then, using (3.26) and (3.29), it is not difficult to get

$$
C_{*} \iint_{Q_{t}}\left|\dot{\theta}_{n}\right|^{2} d \mathbf{x} d s+k\left\|\nabla \theta_{n}(\cdot, t)\right\|^{2} \leq C_{1}+C_{2} \iint_{Q_{t}}\left|\theta_{n}\right|^{2} d \mathbf{x} d s
$$

for all $t \in[0, T]$. Since, by the Hölder inequality,

$$
\begin{aligned}
& \iint_{Q_{t}}\left|\theta_{n}\right|^{2} d \mathbf{x} d s \leq \int_{0}^{t}\left\|\theta_{0}+\int_{0}^{s} \dot{\theta}_{n}(\cdot, \sigma) d \sigma\right\|^{2} d s \\
& \quad \leq 2 t\left\|\theta_{0}\right\|^{2}+2 \int_{0}^{t} s \int_{0}^{s}\left\|\dot{\theta}_{n}(\cdot, \sigma)\right\|^{2} d \sigma \leq C_{3}+C_{4} \int_{0}^{t}\left(\iint_{Q_{s}}\left|\dot{\theta}_{n}\right|^{2} d \mathbf{x} d \sigma\right) d s,
\end{aligned}
$$

one can handle the last term in (3.30) and exploit the Gronwall lemma to deduce that the left hand sides of (3.30) and (consequently) of (3.29) are bounded independently of $n$. Hence, the same property holds for $\iint_{Q_{t}}\left|\Delta \theta_{n}\right|^{2} d \mathbf{x} d s$, due to (3.28), (3.11) and (3.8). Therefore, we remind (3.18-19) and claim that

$$
\left\|\theta_{n}\right\|_{H^{1}\left(0, T ; L^{2}(\Omega)\right) \cap L^{2}\left(0, T ; H^{2}(\Omega)\right)}+\left\|\chi_{n}\right\|_{H^{1}\left(0, T ; L^{2}(\Omega)\right)} \leq C
$$

for any $n \in \mathbb{N}$. The estimate (3.31) allows us to pass to the limit as $n \nearrow \infty$ by weak compactness, in principle for subsequences. If we prove that the limit of $\left(\theta_{n}, \chi_{n}\right)$ 
solves problem $(\mathrm{P})$, then the uniqueness of the solution ensure the convergence of the whole sequences.

Thus, let $\theta$ and $\chi$ be the weak limits of $\theta_{n}$ and $\chi_{n}$, namely

$$
\begin{aligned}
& \theta_{n} \rightarrow \theta \quad \text { weakly in } H^{1}\left(0, T ; L^{2}(\Omega)\right) \cap L^{2}\left(0, T ; H^{2}(\Omega)\right), \\
& \chi_{n} \rightarrow \chi \quad \text { weakly in } H^{1}\left(0, T ; L^{2}(\Omega)\right),
\end{aligned}
$$

as $n \nearrow \infty$. Now, (3.32) and a standard compact embedding yield

$$
\theta_{n} \rightarrow \theta \quad \text { strongly in } L^{2}\left(0, T ; L^{2}(\Omega)\right) \equiv L^{2}(Q)
$$

On the other hand, we would need a strong convergence also for $\chi_{n}$, to deal with the nonlinearities of (3.27-28). Note that

$$
\chi_{n} \rightarrow \chi \quad \text { strongly in } L^{2}(Q)
$$

cannot be inferred from (3.33). We show (3.35) by directly checking that $\left\{\chi_{n}\right\}$ is a Cauchy sequence in $C^{0}\left([0, T] ; L^{2}(\Omega)\right)$. Writing $(3.27)$ for another index $m$ and arguing as in the proof of (3.25), by (3.26) one can easily obtain

$$
\begin{array}{r}
\frac{1}{2}\left\|\left(\chi_{n}-\chi_{m}\right)(\cdot, t)\right\|^{2} \leq \frac{1}{2} \iint_{Q_{t}}\left|\theta_{n}-\theta_{m}\right|^{2} d \mathbf{x} d s \\
+C \iint_{Q_{t}}\left(\left|\theta_{n}\right|^{2}+1\right)\left|\chi_{n}-\chi_{m}\right|^{2} d \mathbf{x} d s .
\end{array}
$$

Moreover, thanks to the embedding $H^{2}(\Omega) \subseteq L^{\infty}(\Omega)$ and the Gronwall lemma, from (3.36) we conclude that

$$
\left\|\left(\chi_{n}-\chi_{m}\right)(\cdot, t)\right\|^{2} \leq\left\|\theta_{n}-\theta_{m}\right\|_{L^{2}(Q)}^{2} \exp \left(C\left(T+\left\|\theta_{n}\right\|_{L^{2}\left(0, T ; H^{2}(\Omega)\right)}^{2}\right)\right)
$$

for any $t \in[0, T]$. Then, due to (3.34) and $(3.31),\left\|\left(\chi_{n}-\chi_{m}\right)\right\|_{C^{0}\left([0, T] ; L^{2}(\Omega)\right)}$ tends to 0 as $n, m \nearrow \infty$ and (3.35) follows.

At this point, it is not difficult to verify that $\theta$ and $\chi$ fulfil (3.13-19). The property $\theta \in C^{0}\left([0, T] ; H^{1}(\Omega)\right)$ is a consequence of the other inclusions, and (3.16), (3.18-19) are readily deduced from (3.32-33). In view of (3.7-8), (3.11-12) and (3.23), the convergences (3.34-35) and the Lipschitz continuity of the involved functions allow us to infer that $A\left(\theta_{n}\right) \rightarrow A(\theta), F_{1}^{\prime}\left(\chi_{n}\right) \rightarrow F_{1}^{\prime}(\chi), 1 / N\left(\theta_{n}\right) \rightarrow 1 / N(\theta)$, $\beta_{1}\left(\chi_{n}\right) \rightarrow \beta_{1}(\chi), \beta_{2}\left(\chi_{n}\right) \rightarrow \beta_{2}(\chi)$ strongly in $L^{2}(Q)$ as $n \nearrow \infty$. In addition, by (3.31) and (3.11) we have that $A\left(\theta_{n}\right) \rightarrow A(\theta)$ weakly in $H^{1}\left(0, T ; L^{2}(\Omega)\right)$, whence

$$
\frac{\partial}{\partial t} A\left(\theta_{n}\right)=\alpha\left(\theta_{n}\right) \dot{\theta}_{n} \rightarrow \frac{\partial}{\partial t} A(\theta)=\alpha(\theta) \dot{\theta} \quad \text { weakly in } L^{2}(Q) \text {. }
$$

Thus, observing that

$$
F_{1}^{\prime}\left(\chi_{n}\right) \dot{\chi}_{n} \rightarrow F_{1}^{\prime}(\chi) \dot{\chi} \quad \text { weakly in } L^{1}(Q)
$$


taking the limit in $(3.28)$ we obtain (3.15). To pass to the limit in (3.27), we point out that, analogously, $\dot{\chi}_{n}\left(\chi_{n}-\xi\right) \rightarrow \dot{\chi}(\chi-\xi)$ weakly in $L^{1}(Q)$ and that inequalities are preserved by the weak limit. Moreover, the right hand side of (3.27) converges to the right hand side of (3.17) strongly in $L^{1}(Q)$. Indeed, by (3.7), (3.12) and (3.16) it is straightforward to realize that

$$
\frac{\beta_{1}\left(\chi_{n}\right)}{N\left(\theta_{n}\right)}\left(\chi_{n}-\xi\right) \rightarrow \frac{\beta_{1}(\chi)}{N(\theta)}(\chi-\xi) \text { strongly in } L^{2}(Q),
$$

while the analogous property for $\tau_{n}\left(\theta_{n}\right)$ may be checked via the inequalities

$$
\begin{aligned}
\iint_{Q}\left|\tau_{n}\left(\theta_{n}\right)-\theta\right|^{2} d \mathbf{x} d t & \leq 2 \iint_{Q}\left(\left|\tau_{n}\left(\theta_{n}\right)-\tau_{n}(\theta)\right|^{2}+\left|\tau_{n}(\theta)-\theta\right|^{2}\right) d \mathbf{x} d t \\
& \leq 2 \iint_{Q}\left|\theta_{n}-\theta\right|^{2} d \mathbf{x} d t+2 \iint_{\{|\theta|>n\}}|\theta|^{2} d \mathbf{x} d t .
\end{aligned}
$$

Then, recalling Subsection 3.2, it is clear that Theorem 3.1 is completely proved.

\subsection{Boundedness and positivity of temperature}

This last subsection is devoted to the proof of Theorems 3.4 and 3.5.

First, in order to show (3.20), we introduce the set

$$
P:=\{(\mathbf{x}, t) \in Q: 0<\chi(\mathbf{x}, t)<1\},
$$

which is a measurable subset of Q. Due to (3.14) and (3.16), it turns out that $\dot{\chi}=0$ a.e. in $Q \backslash P$, since $Q \backslash P=\{\chi=0\} \cup\{\chi=1\}$ and $\chi$ assumes a constant value in each of the last two sets. On the other hand, if $(\mathbf{x}, t)$ is a point in $P$ such that inequality (3.17) holds, we can take as a test number in (3.17) either some value $\xi>\chi(\mathbf{x}, s)$ or some $\xi<\chi(\mathbf{x}, s)$. Hence, we deduce that

$$
\dot{\chi}=\frac{1}{N(\theta)}\left(\theta \beta_{1}(\chi)-\theta_{c} \beta_{2}(\chi)\right) \quad \text { a.e. in } P .
$$

Let us collect the above remarks in the (almost everywhere) equality

$$
\dot{\chi}(\mathbf{x}, t)= \begin{cases}\left(\left(\theta \beta_{1}(\chi)-\theta_{c} \beta_{2}(\chi)\right) / N(\theta)\right)(\mathbf{x}, t) & \text { if } \quad(\mathbf{x}, t) \in R \\ 0 & \text { if } \quad(\mathbf{x}, t) \in Q \backslash R\end{cases}
$$

and point out that $\dot{\chi} \in L^{\infty}\left(0, T ; L^{2}(\Omega)\right)$, owing to (3.7), (3.12) and to the same regularity for $\theta$ (cf. (3.13)). Now, by (3.23) and (3.11) we can rewrite (3.15) as

$$
\frac{\partial A(\theta)}{\partial t}-k \Delta \theta=-\theta_{c} F_{1}^{\prime}(\chi) \dot{\chi} \quad \text { a.e. in } Q .
$$

Note that the function $A$ is Lipschitz continuous along with its inverse function $A^{-1}$. Then, if we specify the new variable $v:=A(\theta)$, we infer that $v$ solves the linear equation

$$
\dot{v}-\operatorname{div}\left(\frac{k}{\alpha(\theta)} \nabla v\right)=-\theta_{c} F_{1}^{\prime}(\chi) \dot{\chi} \quad \text { a.e. in } Q,
$$


with right hand side in $L^{\infty}\left(0, T ; L^{2}(\Omega)\right)$. Since

$$
\frac{\partial v}{\partial \mathbf{n}}=0 \quad \text { a.e. in } \Sigma, \quad v(\cdot, 0)=A\left(\theta_{0}\right) \quad \text { a.e. in } \Omega
$$

and $\theta_{0} \in L^{\infty}(\Omega)$, thanks to (3.11) we can apply Theorem III.7.1 of Ref. 16 and conclude that $v \in L^{\infty}(Q)$, whence (3.20) folllows from $\theta=A^{-1}(v)$ and (3.37).

For the proof of (3.21), the idea of the procedure is to test equation (3.15) by the function (cf. (3.13))

$$
\theta^{-}=\max \{0,-\theta\} \in L^{2}\left(0, T ; H^{1}(\Omega)\right)
$$

and try to show that $\theta^{-}=0$ almost everywhere in $Q$. Also here, we make full use of the equality (3.37). Then, let us multiply (3.15) by $-\theta^{-}$and integrate by parts in space and time. In view of (3.11) and (3.18-19), it is not difficult to get

$$
\frac{C_{*}}{2}\left\|\theta^{-}(\cdot, t)\right\|^{2}+k \iint_{Q_{t}}\left|\nabla \theta^{-}\right| d \mathbf{x} d s \leq \frac{C^{*}}{2}\left\|\left(\theta_{0}\right)^{-}\right\|^{2}+\iint_{Q_{t}} \theta_{c} F_{1}^{\prime}(\chi) \dot{\chi} \theta^{-} d \mathbf{x} d s
$$

for any $t \in[0, T]$. As $\left(\theta_{0}\right)^{-}=0$ a.e. in $\Omega$, with the help of (3.37) we deduce that

$$
\frac{C_{*}}{2}\left\|\theta^{-}(\cdot, t)\right\|^{2} \leq \iint_{Q_{t} \cap P} \frac{\theta_{c} F_{1}^{\prime}(\chi) \theta^{-}}{N(\theta)}\left(\theta \beta_{1}(\chi)-\theta_{c} \beta_{2}(\chi)\right) d \mathbf{x} d s
$$

and consequently

$$
\frac{C_{*}}{2}\left\|\theta^{-}(\cdot, t)\right\|^{2} \leq C \iint_{Q_{t}}\left|\theta \theta^{-}\right| d \mathbf{x} d s-\iint_{Q_{t} \cap P} \frac{\theta_{c}^{2} F_{1}^{\prime}(\chi) \beta_{2}(\chi) \theta^{-}}{N(\theta)} d \mathbf{x} d s,
$$

because of the boundedness of the functions $1 / N, F_{1}^{\prime}, \beta_{1}$. But one sees that $\left|\theta \theta^{-}\right|=$ $\left(\theta^{-}\right)^{2}$ and $\theta^{-} \geq 0$, thus (3.7), (3.9) and (3.12) allow us to obtain

$$
\frac{C_{*}}{2}\left\|\theta^{-}(\cdot, t)\right\|^{2} \leq C \int_{0}^{t}\left\|\theta^{-}(\cdot, s)\right\|^{2} d s
$$

for all $t \in[0, T]$. At this point, it suffices to apply the Gronwall lemma achieving $\theta^{-}=0$ a.e. in $Q$, that entails (3.21).

\section{Acknowledgment}

This work was done under the auspices of GNAFA \& GNFM - CNR and was partially supported by the Italian MURST through the project "Metodi Matematici nella Meccanica dei Sistemi Continui". The first author also benefited from the financial support and kind hospitality provided by the Mathematics Department of the University of Torino.

\section{References}

1. S. M. Allen and J. W. Cahn, A microscopic theory for antiphase motion and its application to antiphase domain coarsening, Acta Metall. 27 (1979) 1085-1095. 
2. D. Blanchard, A. Damlamian and H. Guidouche, A nonlinear system for phase change with dissipation, Differential Integral Equations 2 (1989) 344-362.

3. D. Blanchard and H. Guidouche, A nonlinear system for irreversible phase changes, European J. Appl. Math. 1 (1990) 91-100.

4. H. Brézis, Opérateurs maximaux monotones et semi-groupes de contractions dans les espaces de Hilbert, North-Holland Math. Stud., Vol. 5 (North-Holland, 1973).

5. M. Brokate and J. Sprekels, Hysteresis and phase transitions, Appl. Math. Sci., Vol. 121 (Springer, 1996).

6. G. Caginalp, An analysis of a phase field model of a free boundary, Arch. Rational Mech. Anal. 92 (1986) 205-245.

7. J. W. Cahn and J. E. Hilliard, Free energy of a non uniform system. I. Interfacial free energy, J. Chem. Phys. 28 (1957) 258-267.

8. P. Colli and K. H. Hoffmann, A nonlinear evolution problem describing multi-component phase changes with dissipation, Numer. Funct. Anal. Optim. 14 (1993) 275-297.

9. P. Colli and J. Sprekels, On a Penrose-Fife model with zero interfacial energy leading to a phase-field system of relaxed Stefan type, Ann. Mat. Pura Appl. (4) 169 (1995) 269-289.

10. P. Colli and J. Sprekels, Global solution to the Penrose-Fife phase-field model with zero interfacial energy and Fourier law, Adv. Math. Sci. Appl., to appear.

11. A. Damlamian, N. Kenmochi and N. Sato, Subdifferential operator approach to a class of nonlinear systems for Stefan problems with phase relaxation, Nonlinear Anal. 23 (1994) 115-142.

12. M. Fabrizio and G. Gentili On thermodynamics of some phase change models, Rend. Sem. Mat. Fis. Milano, to appear.

13. M. Fabrizio and C. Giorgi, Sulla termodinamica dei materiali semplici, Boll. Un. Mat. Ital. $B(6) 5$ (1986) 441-464.

14. G. J. Fix, Phase field models for free boundary problems, in Free boundary problems: theory and applications. Vol. II, eds. A. Fasano and M. Primicerio, Pitman Res. Notes Math. Ser., Vol. 79, pp. 580-589 (Longman, 1983).

15. M. Frémond and A. Visintin, Dissipation dans le changement de phase. Surfusion. Changement de phase irréversible, C. R. Acad. Sci. Paris Sér. II Méc. Phys. Chim. Sci. Univers Sci. Terre 301 (1985) 1265-1268.

16. O.A. Ladyženskaja, V.A. Solonnikov and N.N. Ural'ceva, Linear and quasilinear equations of parabolic type, Trans. Amer. Math. Soc., Vol. 23, (Amer. Math. Soc., 1968).

17. O. Penrose and P. C. Fife, Thermodynamically consistent models of phase-field type for the kinetics of phase transitions, Physica D 43 (1990) 44-62.

18. O. Penrose and P. C. Fife, On the relation between the standard phase-field model and a "thermodynamically consistent" phase-field model, Physica D 69 (1993) 107-113.

19. J. Shirohzu, N. Sato and N. Kenmochi, Asymptotic convergence in models for phase change problems, in Nonlinear analysis and applications, eds. N. Kenmochi, M. Niezgódka and P. Strzelecki, GAKUTO Internat. Ser. Math. Sci. Appl., Vol. 7, pp. 361-385 (Gakkotosho, 1995).

20. A. Visintin, Stefan problem with phase relaxation, IMA J. Appl. Math. 34 (1985) $225-245$

21. A. Visintin, Supercooling and superheating effects in phase transitions, IMA J. Appl. Math. 35 (1985) 233-256.

22. A. Visintin, On supercooling and superheating effects in phase transitions, Boll. Un. Mat. Ital. C (6) 5 (1986) 293-311.

23. A. Visintin, Models of phase transitions, Progr. Nonlinear Differential Equations Appl., Vol. 28 (Birkhäuser, 1996).

24. A. A. Wheeler and G. B. McFadden, $A$-vector formulation of anisotropic phase-field models: 3D asymptotics, European J. Appl. Math. 7 (1996), 367-381. 